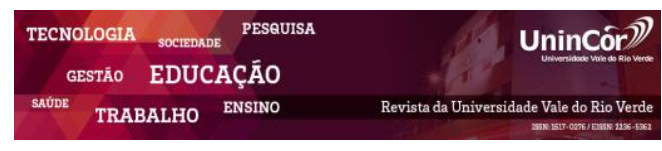

Revista da Universidade Vale do Rio Verde ISSN: 1517-0276 / EISSN: 2236-5362 v. $16\left|\mathbf{n}^{\circ} .3\right|$ Ano 2018

Elaine Aparecida Rocha DOMINGUES Professora e Coordenadora do Curso de Enfermagem

Universidade Vale do Rio Verde (UninCor) elainerocha.contato@gmail.com

Eliana Gomes de NORONHA Acadêmica do Curso de Graduação em Enfermagem.

Universidade Vale do Rio Verde (UninCor) elianasaothome@hotmail.com email@email.com

Maiúme Roana Ferreira de CARVALHO

Enfermeira, Mestre em Ciências da Saúde e Supervisora de Estágio do curso de Enfermagem

Universidade Vale do Rio Verde (UninCor) enf_maiume@yahoo.com.br

Glei dos Anjos de CARVALHO-CASTRO Professora Doutora em Ciências da Universidade do Vale do Rio Verde (UninCor) gleicarv@yahoo.com.br

\section{FATORES ESTRESSANTES VIVENCIADOS PELOS PAIS DE CRIANÇAS COM DEFICIÊNCIA EM TRÊS CORAÇÕES-MG}

\section{RESUMO}

A educação de uma criança com deficiência leva os tutores a passar por diferentes situações e desequilíbrio emocional. O objetivo deste trabalho foi levantar fatores estressantes para tutores de crianças com deficiência. Foi realizada uma pesquisa descritiva, transversal, com abordagem qualitativa, na Associação de pais e amigos excepcionais (APAE) de Três Corações - MG. A coleta de dados ocorreu de outubro a novembro de 2017 por meio de entrevista semiestruturada e analise foi realizada por meio do Discurso do Sujeito Coletivo. A população estudada encontrava-se na faixa etária de 20 a 40 anos, maioria casado, com renda familiar predominante de um a três salários mínimos relacionado a um baixo nível de escolaridade. A análise deste estudo demonstrou que os principais fatores estressantes vivenciados pelos entrevistados foram o transporte das crianças, o preconceito das pessoas, o medo e insegurança. A identificação desses fatores é necessária para o enfrentamento dos tutores a situação.

Palavras-chave: APAE; transporte; preconceito; medo; insegurança.

\section{STRESSING FACTORS EXPERIENCED BY PARENTS OF DISABLED CHILDREN}

\begin{abstract}
The education of a disabled child leads tutors to different situations and emotional imbalance. The objective of this work was to raise stressors for tutors of children with disabilities. An exploratory, descriptive, cross - sectional study with a qualitative approach was carried out at the Association of Expecting Parents and Friends (AEPF) of Três Corações - MG. The date collection was performed from October to November of 2017 through a semi-structured and analyzed interview of the Discourse of the Collective Subject. The population studied was in the age group of 20 to 40 years, most married, with a predominant family income of one to three minimum wages related to a low level of schooling. The analysis of this study showed that the main stressors experienced by the interviewees were children's transportation, people's prejudgement, fear and insecurity.

Keywords: Expecting Parents; children's transportation; people's prejudgement; fear; insecurity.
\end{abstract}




\section{INTRODUÇÃO}

A gestação planejada gera na família uma grande expectativa, o casal idealiza a criança e elabora planos para seu futuro. (GOMES, 2007). $\mathrm{O}$ nascimento de uma criança gera sentimentos de dúvidas e ansiedade em relação ao futuro, o que é exacerbado quando os progenitores são informados que terão uma criança com deficiência. Consequentemente, os pais enfrentam períodos difíceis devido às frustrações e desequilíbrio emocional que vivenciam e passam por um período de luto, ou seja morreu o filho idealizado (THUY ,BERRY, 2013; BRITO, DESSEN, 1999). Apesar da rejeição inicial, devido as limitações enfrentadas pela criança, ela se torna superprotegida (AUGUSTO, CHACON, 2011; CUNHA, 2010).

O desenvolvimento das crianças, independente de ser especial ou não, tem a influência individual associada às necessidades distintas sem a influência do diagnóstico. Porém, no caso das crianças especiais é crucial que nos primeiros anos de vida se inicie o tratamento adequado juntamente com as orientações aos pais. Além disso, a inserção em um ambiente acolhedor permite o desenvolvimento de forma integral.

Muitas vezes este desenvolvimento de forma integral é dificultado devido a falta de conhecimento da sociedade que vê a deficiência como uma doença crônica sem possibilidade de alteração no quadro clínico. Isto leva, muitas vezes, ao tratamento de pessoas deficientes como seres incapazes, sem direitos e excluídos. Esta situação pode se agravar em famílias de baixa renda, pois a falta de recursos não os permite a realização de um tratamento adequado (SANTOS E OLIVEIRA, 2015). Para pais de baixa renda a APAE é uma grande aliada.

A Associação de Pais e Amigos dos Excepcionais foi criada em 1954, no Rio de Janeiro. Trata-se de uma organização social que visa a promoção da atenção integral à pessoa com deficiência. Esta associação se encontra em mais de 2 mil municípios do território nacional.

Diversas ferramentas são utilizadas para levantamento de dados qualitativos, dentre elas o Discurso do Sujeito Coletivo (DSC) que funciona como um método de resgate da Representação Social (RS) caracterizado pelo reconstituir dados individuais com uma visão coletiva (LEFÈVRE; LEFÈVRE, 2014)

Apesar de estarmos na era da acessibilidade a informações, não há dados sobre os problemas enfrentados pelos pais de crianças excepcionais, estudantes, da APAE de Três Corações- MG. Diante deste cenário, o objetivo deste estudo foi conhecer os fatores estressantes vivenciados pelos progenitores de estudantes da APAE de Três Corações - MG.

\section{MATERIAL E MÉTODOS}

O presente trabalho é do tipo descritivo, transversal, com foco na abordagem qualitativa e fundamentado na análise do Discurso do Sujeito Coletivo. Ele foi realizado na cidade de Três 
Corações- MG, localizado no sul de Minas Gerais. O questionário foi aplicado aos pais da Associação de Pais e Amigos Excepcionais (APAE), localizado a Avenida Deputado Renato Azeredo em Três Corações. Esta escola é destinada ao atendimento integral de crianças portadoras de necessidades especiais.

Adotou-se a entrevista semiestruturada como técnica de coleta de dados. A seleção dos sujeitos foi efetuada por meio da indicação da psicóloga e assistente social pela listagem com os nomes dos progenitores, os quais se incluíam nos seguintes critérios: ser pais de crianças com diagnóstico médico de deficiência e que frequentam a APAE e como excluídos os pais de criança que não tinham um diagnóstico médico definitivo associado aqueles que não aceitaram participar do estudo.

Para a coleta de dados, que foi realizada no período de outubro a novembro de 2017 , foi utilizado um gravador de voz digital, em que os depoimentos buscando a relação das dificuldades e caracterização sóciodemográfica dos entrevistados. Todas as entrevistas foram gravadas mediante a autorização dos responsáveis, em local e horário previamente agendado, permitindo uma maior privacidade aos participantes.

Após a coleta de dados, foi realizada a transcrição e leitura minuciosa do material empírico e posteriormente analisados por meio da técnica do Discurso do Sujeito Coletivo (DSC) de acordo com Lefèvre (2005). Para a elaboração do Discurso do Sujeito Coletivo (DSC), os discursos brutos de cada pesquisado foram submetidos a um trabalho analítico inicial de decomposição que consistiu na seleção das expressões-chave (ECH) e Ideias centrais (IC) de cada discurso individual. Após, todos eles foram reunidos, buscando a representação social. As expressões-chave (ECH) são segmentos de discursos que remetem a uma ideia central (IC) presente neste discurso. A ideia central (IC) tem a importante função de individualizar um dado discurso ou conjunto de discursos, descrevendo suas especificidades semânticas.

Todos os entrevistados assinaram o Termo de Consentimento Livre e Esclarecido (TCLE), segundo Resolução No 466/12 do Conselho Nacional de Saúde. Garantindo o sigilo das informações obtidas individualmente e a liberdade para se recusarem ou desistirem da pesquisa, em qualquer momento, sem que isso pudesse causar-lhe algum prejuízo. O estudo foi realizado após aprovação pelo Comitê de Ética em Pesquisa (CEP) da Universidade Vale do Rio Verde - UninCor.

\section{RESULTADOS E DISCUSSÃO}

Apenas dez (10) pais, dos indicados pela assistente social e psicóloga da APAE - Três Corações, aceitaram participar da pesquisa. Isto era esperado uma vez que muitos pais sentem dificuldades para falar sobre a deficiência dos filhos com pessoas estranhas.

Em relação aos aspectos sócios demográficos dos entrevistados (tabela1), observou-se que eles se enquadravam principalmente na faixa etária entre 25 a 35 anos, eram casados com renda familiar de um a três salários mínimos e nível de escolaridade ensino fundamental completo ou incompleto. No que se refere a número de filhos as progenitoras obtiveram uma média de 2 filhos e as crianças 
deficientes tinham em média 08 anos no momento da realização do trabalho. Quanto ao histórico familiar de deficiência, prevaleceu a não ocorrência em $90 \%$ das famílias entrevistadas.

Após a análise do material coletado, as Ideias Centrais e os receptivos Discurso do Sujeito Coletivo, referente a temática que emergiram provenientes da transcrição e interpretação das falas dos entrevistados foram organizados. Tivemos como resultado que alguns pais com certeza enfrentam limitações (Sim), outros às vezes enquanto que para alguns não há problemas enfrentados (não). A diferença no ponto de vista da presença ou ausência de limitações apresentadas pode estar relacionada à fase de vivência com a deficiência em que a família se encontra. Estas, segundo dados prévios são três: o momento de choque em que a família passa por situações como ansiedade, impotência, culpa e negação; a fase da aceitação em que já sabem identificar e organizar ideias sobre a deficiência; e finalmente não demonstram seus sentimentos, mesmo que eles existam(COSTA, 2012). Do ponto de vista prático, quanto mais rápido for a passagem da família por estas fases, maior será a capacidade de inserção da criança na sociedade. Isto devido o início precoce com tratamentos e alternativas que possibilitem uma redução dos sintomas limitadores. Ademais, isto possibilita um melhor controle sobre aqueles sentimentos gerados pelo diagnóstico e informação que serão pais de crianças especiais.

Em presença das considerações feitas pelos sujeitos da pesquisa, os mesmos foram convidados para melhor elucidar sobre a existência de limitação pelo fato de possuir um filho especial, e emergiram as seguintes ideias centrais e o referido discurso DSC: "A dificuldade que ela tem é ir para a escola... é ter o cuidado com as outras crianças... o preconceito das pessoas de olhar aquela criança, do jeito que ele é, das pessoas na rua, preconceito com as outras crianças com ela, não quer brincar, quer ficar distante... tenho medo de cair, pois onde moro tem escada de 13 degraus... receio no começo, será que vou conseguir?..." As palavras chaves encontradas no discurso estão representadas na figura 1 , sendo que três delas: cuidado, insegurança e medo estão diretamente relacionadas ao sentimento de impotência dos pais para enfrentar a situação. Além disso, fica subentendido a alta dependência desta criança em relação aos pais. Esta característica foi relatada em outros trabalhos que mostram a alta demanda de tempo dos pais pelos filhos com deficiência (CAROTENUTO, M et al., 2017).

Estes sentimentos incertos que surgem estão relacionados a idealização da família durante a gestação, e o enfrentamento define a vivência da criança deficiente com seus familiares e a sociedade (BROWNE; MILLAR, 2016; SANTOS E OLIVEIRA, 2015; DUARTE, 2010). O sentimento de impotência relatado pelos entrevistados é um fator comum entre pais de crianças com deficiência. Esta característica é na maioria das vezes causa de sofrimento, desconforto, constrangimento, tristeza e confusão para todos os integrantes da família, adicionalmente exigem maior tempo disponível além da insegurança e sentimento de solidão (SANCHES, JUNIOR, 2011; BUSCAGLIA, 2002; BARBOSA MAM, 2000) . 
O transporte, outra ideia central relatada, foi encontrado na seguinte expressão chave" Para poder sair de casa com ele né, e ele usa fralda, tem a parte de transporte também, porque as vezes tem muita gente que não reconhece que a criança as vezes precisa de por exemplo de sentar no ônibus, no ônibus ter lugar para poder que a criança sentar, as vezes não sede lugar”. Este fator está associado à condição social que se enquadra os entrevistados uma vez que este grupo depende mais das políticas públicas. Este relato está de acordo com o que foi mostrado por Castro, Cieza, Cezar, (2011) que as pessoas portadoras de necessidades especiais enfrentam mais barreiras geográficas, comportamentais financeiras e outras. Entretanto, vale ressaltar que o grau de aceitação do indivíduo deficiente na sociedade não apresenta relação com recursos financeiros (MUNYI, 2012).

Adicionalmente, vale destacar que além do transporte tem toda a preparação de ida e vinda a unidades de saúde, os horários de atendimentos rígidos (que para os pais dependentes do transporte público é um gargalo maior ainda). Ainda tem para aqueles com veículo próprio a dificuldade de estacionar e de mobilidade dentro dos serviços de saúde, e muitas vezes se deparam com profissionais de saúde sem conhecimento sobre a deficiência (WHARTON, HAMES, MILNET, 2011).

Este entendimento da deficiência às vezes é um obstáculo dentro do próprio ambiente familiar e está vinculado a falta de conhecimento da sociedade (SANTOS E OLIVEIRA, 2015). Assim como uma herança cultural do perfeccionismo, o que leva pessoas especiais serem tratadas como incapazes, sem direitos e esquecidos pela sociedade . Frente a este cenário projetos de educação em saúde relacionados as deficiências físicas e mentais são necessários para auxiliar e orientar os progenitores no enfrentamento a condição de ter um filho deficiente.

\section{CONCLUSÃO}

Com este trabalho pode se identificar os principais fatores estressantes para pais das crianças de APAE de Três Corações-MG. Este funcionará como embasamento para estratégias de saúde pública visando o auxilio no enfrentamento desta situação e aliviar o desconforto vivido por estas pessoas. 
Figura 1. Ideias Centrais e Discurso do Sujeito Coletivo referente as limitações frente a um filho especial, Três Corações. MG. $(\mathrm{n}=10)$

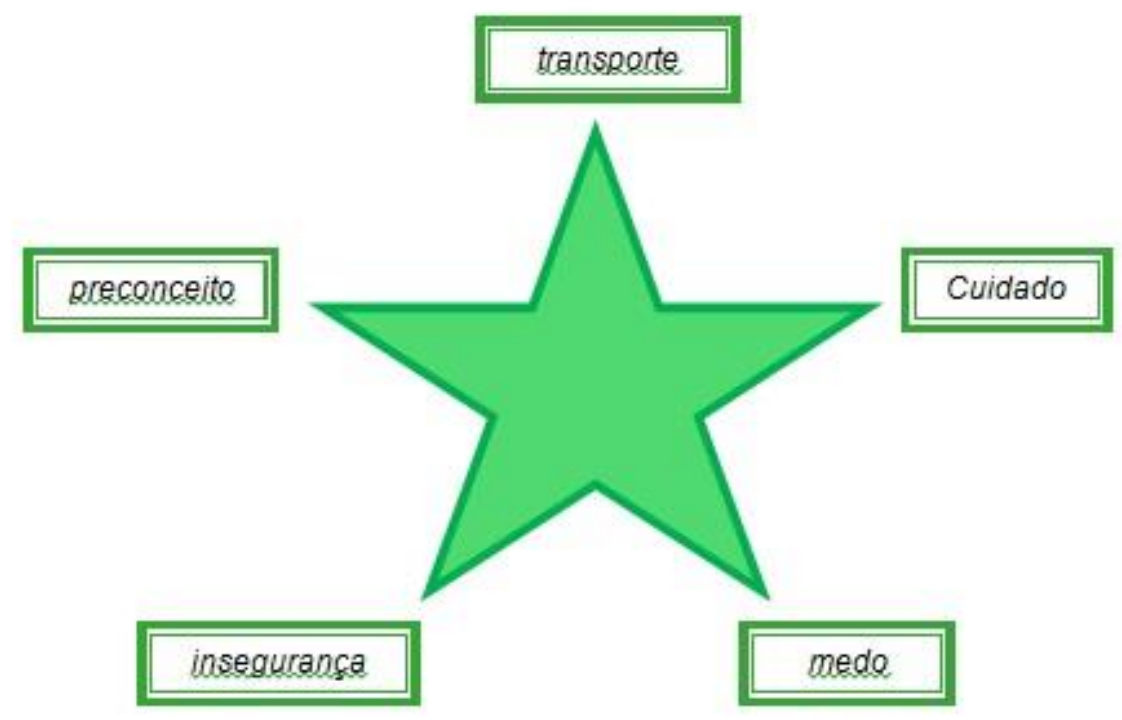

Tabela 1. Distribuição das características sóciodemográficas dos progenitores entrevistados na APAE de Três Corações MG. $(\mathrm{n}=10)$

\begin{tabular}{lll}
\hline \multicolumn{1}{c}{ Características } & N & \% \\
\hline Faixa etária(anos) & & \\
$15-25$ & 02 & 20 \\
$\mathbf{2 5 - 3 5}$ & $\mathbf{0 6}$ & $\mathbf{6 0}$ \\
$>35$ & 02 & 20 \\
Casada & $\mathbf{0 7}$ & $\mathbf{7 0}$ \\
\hline Solteiro & 02 & 20 \\
Divorciado & 01 & 10 \\
\hline Renda Familiar & & \\
\hline 1 a 3 salários mínimos & $\mathbf{1 0}$ & $\mathbf{1 0 0}$ \\
\hline Escolaridade & & \\
Fundamental & $\mathbf{0 5}$ & $\mathbf{5 0}$ \\
incompleto & & \\
Fundamental completo & $\mathbf{0 5}$ & $\mathbf{5 0}$ \\
História familiar & & \\
Sim & 01 & 10 \\
Não & 09 & 90 \\
\hline
\end{tabular}




\section{REFERÊNCIAS}

AUGUSTO, M.A.P.C; CHACON,M.C. M.L,

Diferentes enfoques das relações familiares: superproteção e abandono. In: Congresso Multidisciplinar Universidade Estadual de Londrina. p.1316-1327, 2011.

BARBOSA MAM. Compreendendo o mundo-vida da mãe com um filho deficiente[tese]. São

Paulo: Universidade federal de São Paulo.

Departamento de Enfermagem; 2000.

BRITO AMW, Dessen MA. Crianças surdas e suas famílias: um panorama geral. Psicol Reflex Crit, v.12, n.2, p. 429-45.1999.

BROWNE, M. ; MILLAR, M. . A rights-based conceptual framework for the social inclusion of children and young persons with an intellectual disability, Disability \& Society, v.31, n.8,p. 10641080, 2016.

BUSCAGLIA L. Os e seus pais: Um desafio ao aconselhamento. $4^{\mathrm{a}}$ ed. Rio de Janeiro: record; 2002.

CAROTENUTO M, MESSINA A, MONDA V, et al..Maternal Stress and Coping Strategies in Developmental Dyslexia: An Italian Multicenter Study.Front Psychiatry. V.22;8:295, 2017; doi:10.3389/fpsyt.2017.00295. e Collection 2017.

CASTRO SS, CIEZA A, CESAR CL. Problems with accessebility to health services by persons with disabilities in São Paulo, Brazil. Disabil Rehabil. v.33, n.17-18, p.1693-8, 2011.

COSTA, S.C.P. O impacto do diagnóstico de autismo nos pais. Universidade Católica Portuguesa Centro Regional da Beiras. Viseu, 2012. Disponível em: http://repositorio.ucp.pt/bitstream/10400.14/11964/1/ O\%20impacto\%20do\%20diagn\%C3\%B3stico\%20de $\%$ 20autismo\%20nos\%20pais.pdf Acesso em: 13 No. 2016.

CUNHA. M. A. F. V.; BLASCOVI-ASSIS, S. M FIAMENGUI, G.A. Impacto da notícia da síndrome de Down para os pais: história de vida. Ciência e Saúde Coletiva, v.15, n.2, p. 444-451, 2010.

DUARTE, M, R. Percepções, Sentimentos e Receios de Famílias de Crianças com Deficiência: Um Estudo de Casos Múltiplos.

Universidade de Coimbra Faculdade De Psicologia e Ciências da Educação. Coimbra, 2010.

GOMES, Aline Grill; Mal formação do bebê e maternidade: impacto de uma psicoterapia breve paisbebê para as representações da

mãe; Universidade federal rio grande do sul, porto alegre, agosto 2007.

LEFÈVRE, F.; LEFÈVRE, A. N. M. Texto Contexto Enferm, Florianópolis, Abr-Jun; v.23 n.2, p. 502-507, 2014.
LEFÈVRE, F.; LEFÈVRE, A. N. M. Discurso do sujeito coletivo: um novo enfoque em pesquisa qualitativa (desdobramentos). 2 ed. Caxias do Sul:EDUCS, 2005.

MUNYI, C. W. (2012). Past and present perceptions towards disability: A historical perspective. Disability Studies Quarterly, 32(2). Retrieved from http://dsqsds.org/article/view/3197/3068.

PETEAN, E.B.L, PINA-NETO, J.M. Investigação em aconselhamento genético: impacto da primeira noticia_a reação dos pais à deficiência. Medicina: Ribeirão Preto, v.31, n.2 p. 288-295, 1998.

PINTO, M. B. et al. Significado do cuidado à criança deficiente com necessidade especiais: relato de mães. Revista Ciência Cuidado e Saúde. v.13 n.3, p.549-555, Jul./Set, 2014. Disponível em:

http://www.periodicos.uem.br/ojs/index.php/CiencCui dSaude/article/viewFile/22189/pdf_228 Acesso em 13 Nov. 2016.

SANCHES L.A.S, JUNIOR G.AF. Relatos maternos sobre o impacto do diagnostico da deficiência dos filhos. Cad. Saúde. Colet., Rio de janeiro, v.19, n.3, p 366-74.2011. Disponível em:

http://www.iesc.ufrj.br/cadernos/imagens/csc/2011 3/ artigos/csc v19n3366-374.pdf.> Acesso: 05 Maio de 2016.

SANTOS,A.C.A., OLIVEIRA, V.M.S. A família como elemento para a inclusão social do deficiente. Ideias e Inovação, Aracaju. v. 2, n. 2, p. 47-48, Março, 2015.

SOUSA,R,C. Reações e expectativas de mães em relação à surdez de seus filhos. Universidade Estadual de Ceará. Centro de ciências da saúde, Curso de mestrado profissional em saúde da criança e do adolescente. Fortaleza- Ceará 2011.

SOUZA, L.G.A. Cuidando do filho com deficiência mental: desvelamento de vivencias de pais no seu sercom-o-filho[tese]. Ribeirão Preto: Escola de enfermagem de Ribeirão Preto da Universidade de São Paulo; 2003.

THUY, N.T.M.; BERRY, H.L. Social capital and mental health among mothers in Vietnam who have children with disabilities. Glob Health Action. V. 6, p. 1-12, 2013.

WHARTON S, HAMES A, MILNET H. The accebility of general NHS services for children with disabilities. Child care Health \& development. 2005[ cited 2011 Jun 25] 31(3):275-82. Available from: http://www.ncbi.nlm.nih.gov/pubmed/15840147. 


\section{Elaine Aparecida Rocha DOMINGUES}

Professora Doutora pela UNICAMP e Coordenadora do Curso de Enfermagem, Universidade Vale do

Rio Verde (UninCor) de Três Corações (MG).

\section{Eliana Gomes de Noronha}

Acadêmica do Curso de Graduação em Enfermagem. Universidade Vale do Rio Verde (UninCor) Três Corações (MG).

\footnotetext{
Maiúme Roana Ferreira de Carvalho

Enfermeira, Mestre em Ciências da Saúde e Supervisora de Estágio do curso de Enfermagem, Universidade Vale do Rio Verde (UninCor) de Três Corações (MG).
}

\section{Glei dos Anjos de Carvalho-Castro}

Médica Veterinária graduada pela Universidade Estadual de Londrina, Mestre e Doutora em Ciências, Docente da Universidade Vale do Rio Verde (UninCor) de Três Corações (MG). 